\title{
Novel Ceramic-Polymer Composite Membranes for the Separation of Liquid Waste
}

\author{
Annual Progress Report \\ Period: 9/15/1999-3/16/2000 \\ Submitted to: \\ U.S Department of Energy \\ by \\ Yoram Cohen \\ Department of Chemical Engineering \\ University of California, Los Angeles \\ Los Angeles, California 90095-1592 \\ Phone: (310) 825-8766 \\ Fax: (310) 477-3868 \\ E-Mail: Yoram@ucloa.edu
}




\section{Novel Ceramic-Polymer Composite Membranes for the Separation of Hazardous Liquid Waste DOE Project DE-FG07-96ER14715 \\ Progress Report Period: 9/15/1999-3/16/2000}

Prepared by

Yoram Cohen, Chemical Engineering

\section{PROJECT SUMMARY}

Department, University of California, Los Angeles

There is a growing need in the areas of hazardous waste treatment, remediation and pollution prevention for new processes capable of selectively separating and removing target organic species from aqueous steams. Membrane separation processes are especially suited for solute removal from dilute solutions. They have the additional advantage of requiring less energy relative to conventional separation technologies (e.g., distillation, extraction and even adsorption processes). The major difficulty with current membranes is the poor longevity of polymeric membranes under harsh conditions (high temperature, harsh solvents and $\mathrm{pH}$ conditions) and the lack of selectivity of ceramic membranes. In our previous work (1996 EMSP project), a first generation of novel polymer-ceramic (PolyCer) composite membranes were developed with the goal $f$ overcoming the above difficulties. The proposed PolyCer membranes are fabricated by a surface-graft polymerization process resulting in a molecular layer of polymer chains which are terminally and covalently anchored to the porous membrane support. The plymer imparts the desired membrane selectivity while the ceramic support provides structural integrity. The PolyCer membrane retain its structural integrity and performance even when the polymer phase is exposed to harsh solvent conditions since the polymer chains are covalently bonded to the ceramic support surface. To date, prototype PolyCer membranes were developed for two different membrane separation processes: (a) pervaporation removal of organics from aqueous systems; and (b) ultrafiltration of oil-in-water emulsions. Pervaporation PolyCer membranes were demonstrated for removal of selected organics (TCE, chloroform and MTBE) from water with permeate enrichment factors as high as 300 . While the above results have been extremely encouraging, higher enrichment factors $(>1000)$ should be sought for field applications. The above improvement is feasible by increasing the length and surface density of the grafted polymer chains. The required simultaneous increase in surface polymer graft density and chain length is beyond the capability of present free-radical graft polymerization methods. Therefore, it is proposed to develop a new approach to synthesizing the grafted polymer membrane phase via "living" free-radical polymerization. This approach should allow controlled growth of the grafted polymer chains while maintaining the advantage of high surface chain density possible with conventional free-radical polymerization. Optimization of the membrane surface layer will be sought by developing fundamental correlation between surface characteristics (e.g., topology, chain length and surface density) and membrane performance. The ability to tailor-design the grafted polymer surface with long polymer chains of a desired surface density is also advantageous in fabricating non-fouling ultrafiltration membranes for colloidal filtration.

Using the same "living" free-radical polymerization technology, as for the pervaporation membranes, ultrafiltration ceramic membranes with terminally anchored surface chains, can be produced to repel colloidal species, thus reducing membrane fouling while increasing permeate rejection. As an outcome of the 1996 EEMSP project, it was discovered that, with sufficiently long surface chains, significant increase in PolyCer UF membrane rejection is possible, especially at high tangential velocities. The fabrication, via "living" free-radical polymerization, and optimization of such non-fouling UF membranes is another goal of the proposed research. It is expected that this project will results in the demonstration of a commercially viable technology for the "tailor design" and optimization of a new class of selective and robust polymer-ceramic (PolyCer) membranes for aqueous waste treatment and water decontamination applications. The proposed PolyCer approach will allow the rapid deployment of "field-ready" and task-specific membranes for recovery and recycle for remediation and pollution prevention applications. 


\section{Overall Progress and Accomplishments for the Period 9/15/96-3/15/2000}

This report summarizes the work progress over the last year of the above referenced project. Membrane separation processes are especially suited for solute removal from dilute solutions. They have the additional advantage of requiring less energy relative to conventional separation technologies (e.g., distillation, extraction and even adsorption processes). The major difficulty with current membranes is the poor longevity of polymeric membranes under harsh conditions (high temperature, harsh solvents and $\mathrm{pH}$ conditions) and the lack of selectivity of ceramic membranes. The objective of the project has been to develop a new class of ceramic-supported polymeric membranes that would overcome the above difficulties and could be tailored-designed for a wide-range of applications in remediation and pollution prevention.

The PolyCer membranes are fabricated by a surface-graft polymerization process resulting in a molecular layer of polymer chains which are terminally and covalently anchored to the porous membrane support. The polymer imparts the desired membrane selectivity while the ceramic support provides structural integrity. The PolyCer membrane retain its structural integrity and performance even when the polymer phase is exposed to harsh solvent conditions since the polymer chains are covalently bonded to the ceramic support surface. Prototype PolyCer membranes were developed for two different membrane separation processes: (a) pervaporation removal of organics from aqueous systems; and (b) ultrafiltration of oil-in-water emulsions. Also, as a result of the project work, we have generated fundamental knowledge in the areas of graft polymerization kinetics, hydrodynamics of terminally anchored copolymer chains and polymer grafted-silica resins

As a recognition of the uniqueness of our membrane development approach, we received the 1997 Outstanding Paper award from the AIChE Separations Division [Castro et al., J. Membrane Sci., 115, 179-190, 1996]. In addition, a patent application for ceramic-supported polymer pervaporation membrane was filed with the US Patent Office with the consent of DOE. To date, the work has resulted in the publication of five journal articles and book chapters (Section 1.2), and 13 conference presentations and invited seminars. Four additional papers are currently in preparation for publication. Two Ph.D. students (Deng-Jeng Jou and Robert Castro) and three M.S. students (Niangjiong Bei, Ron S. Faibish and Hiroyoshi Ohya), whose research was supported in whole or to a significant degree by the above gant, graduated during the grant period. Currently the dissertation research work of two Ph.D. students (Wayne H. Yoshida and Ron S. Faibish), whose work on the DOE project commenced two years ago, is in progress. IT is also noted that highlights of our research accomplishments was recently published in Initiatives in Environmental Technology Investment (Volume 6, Winter 1999; http://www.wpi.org/initiatives).

The success of our membrane synthesis work was made possible by being able to carefully control the graft polymerization process having developed a detailed kinetic model of free-radical graft polymerization. This enabled us to increase the polymer graft yield to a higher level than previously possible. Also, it is now possible to increase the contribution of graft polymerization to the total polymer graft yield, thereby increasing the polymer chains density (Figure 1). The high surface chain density is illustrated by AFM image shown in Fig. 2. The surface chains are polydisperse in size as expected from free-radical polymerization.

The hydrophobicity/hydrophilicity of the grafted surfaces was observed via contact angle measurements as illustrated in Fig. 3. For the hydrophilic PVP brush layer, the contact angle for water decreased, with increasing polymer graft yield, indicating a more hydrophilic surface layer. Spreading of water on the PVP grafted layer is possible since the surface chains can absorb water and swell. In contrast, the PVAC layer is sufficiently dense, even at the lowest graft yield, as indicated in Fig. 3; thus, little variation in contact angle is observed on this hydrophobic surface. 
The surface graft polymerization method, developed as part of this project, proved to be effective in masking surface hydroxyl groups on the surface of inorganic oxides. As a result, surface charge was significantly reduced, over a wide $\mathrm{pH}$ range, and therefore interaction of solutes with surface hydroxyls was essentially eliminated. An example, of the effect of poly(vinylpyrrolidone) on masking of $-\mathrm{OH}$ groups on a zirconia surface is illustrated in Fig. 4. Such a surface is efficient in reducing irreversible fouling when treating oil-in-water microemulsions (which are typically negatively charged). Preliminary studies have shown that irreversible fouling, which can lead to permeability loss in excess of 50-100\% in many cases, is virtually eliminated with the use of a PVP-modified membrane. An additional benefit of the grafted polymer phase is that membrane rejection, for UF, can be increased due to the polymer chains which screen the membrane pore from the emulsion droplets, while allowing the continuous phase to permeate through the membrane. An example of the above behavior is shown in Fig. 5 for ultrafiltration of a synthetic oil-in-water emulsion (decane/octanoic acid/sodium octanoate/isobutanol/water) filtered through tubular native and PVP-modified zirconia membrane under laminar flow condition. Rejection was increased by about a factor of 2 (Fig. 5) with the modified membrane.

In a series of studies with filtration of a commercial cutting oil emulsions through a silica tube membrane (120 nm pore size), we have found that significant improvement in rejection of oil was achieved at a sufficiently high tube-side Reynolds number (Fig. 6). It is believed that the above phenomena is due to deformation of the grafted polymer chains, a well documented chain behavior. Work is currently underway to optimize the grafted chain length in order to exploit the full potential of the above shear-induced behavior for improvement of membrane rejection performance.

The graft polymerization method for membrane modification, developed in our laboratory, has proven successful for producing ceramic-supported polymer pervaporation membranes. We have shown that for pervaporation, the starting pore size for the ceramic support should be no larger than about $500 \AA$. PVAc-grafted silica membranes, for example, were shown to be capable of removing volatile chlorinated organic solvents from water by pervaporation (Figs. 8), while PVP-based membranes were useful for polar/non-polar organic/organic separation and dehydration of organic solvents. These membranes exhibited negligible membrane resistance to solute transport through the membrane. The tube-side boundary-layer resistance was the dominant mass transfer resistance which decreased with increasing tube-side Reynolds numbers. The enrichment factors for the removal of trichloroethylene (TCE) and chloroform and MTBE, using an exploratory Poly(vinylacetate)-silica membrane, was sufficiently high (with permeate enrichment factors up to 300) to enable the recovery of pure organic solutes from solutions of concentrations down to about $20 \mathrm{ppm}$ in a one-stage operation (Figs. 7). Our most recent work has suggested that the separation factor can be increased by increasing the polymer graft density. Improved separation of the PolyCer PVAc pervaporation membrane is expected upon reduction of water permeation flux (Fig. 7). Enrichment factors of approaching and even exceeding 1000 (Fig. 7) are possible with free-radical graft polymerization technology. However, modification to the reaction scheme to incorporate termination blockers will be required.

We have demonstrated that initial selection of the polymer for specific separations can be made based on simple solubility considerations. Accordingly, 2-D solubility parameter map is shown in Fig. 8, where solvent (or solute)-polymer compatibility is indicated by the distance between the polymer and organic points on the map; increased compatibility (or solubility of polymer in the organic liquid) is depicted by smaller distances between a selected pair. For example, Poly(vinyl acetate) (PVAc) is completely soluble in trichloroethylene and insoluble in water; thus, PVAc is a suitable polymer for pervaporation removal of trichloroethylene from 
water. Following the above approach, tubular silica and zirconia membranes were modified with PVAc and PVP to form pervaporation membranes.

\subsection{Expected New Developments}

Development work, to date, has been essentially a "proof of concept" effort focused on pervaporation removal of volatile organics from aqueous systems and ultrafiltration of oil-inwater microemulsions with PolyCer membranes. The PolyCer membrane technology has been shown to be applicable for both UF and pervaporation. We are now $\mathrm{n}$ position to advance the PolyCer membranes to the next level - practical PolyCer membranes. To accomplish this goal goal, membrane performance, with respect to the grafted polymer phase, must be fully correlated and optimized. Such continuing work work is essential for the development of PolyCer membranes for field-scale and commercial applications. Growing interest by industry in the PolyCer membrane concept is encouraging. With additional optimization work, we are confident that PolyCer membranes will emerge to meet the demand for membranes that retain their structural integrity and longevity under harsh conditions while maintaining the desired selectivity and permeate fux. The approach will pave the way for a rapid tailor-design of pervaporation and UF membranes for organic-aqueous separations.

\subsection{Publications in Connection with Project DE-FG07-96ER14715}

\subsubsection{Papers and Book Chapters}

1. Castro, R. P., H. G. Monbouquette and Y. Cohen, "Polyvinylpyrrolidone-Silica Membranes for the Treatment of Oil-in-Water Emulsions"," J. Membrane Science, 115, 179-187 (1996).

2. Faibish R., Menachem Elimelech and Y. Cohen, "Effect of Intraparticle Electrostatic Double Layer Interactions on Permeate Flux Decline in Crossflow Membrane Filtration of Colloidal Suspensions: An Experimental Investigation", J. Colloid and Interface Science, 204, 77-86 (1998).

3. Jou, Jeng-Dung, W. Yoshida and Y. Cohen, "Pervaporation with Ceramic-Supported Polymer Membranes", J. Membrane Sci., 162, 269-284 (1999).

4. Cohen, Y., R. Faibish and M. Rovira, Size Exclusion Chromatography with Graft Polymerized Silica Resins, in "Surface Interactions in Size Exclusion Chromatography, "E. Pefferkorn (Editor) Marcel Dekker, (1999).

3. Cohen, Y., J. Deng-Jeng, W. Yoshida and N. Bei, "Surface Modification of Oxide Surfaces by Graft Polymerization," in "Oxide Surfaces", J. A. Wingrave (editor), Marcel Dekker, in press (1999).

4. Yoshida, W., R. Castro and Y. Cohen, Silylation of Inorganic-Oxides Surfaces: The Role of Surface Water,", in preparation.

5. Faibish, R., Yoshida, W. and Y. Cohen, "Surface Topology and Wettability of Grafted Polymer Surfaces," in preparation.

6. Faibish R., W. Y. Cohen, "Ultrafiltration of Oil-in-Water Microemulsions with Zirconia -Supported Polyvinylpyrrolidone Membrane,", in preparation.

9. Yoshida, W. and Y. Cohen, "Optimization of Silica-Grafted Polymer Pervaporation Membrane," in preparation. 


\subsubsection{Conference and Invited Presentations}

1. Cohen, Y., R. P. Castro, J-D Jou and H. G. Monbouquette, "Ceramic-Supported Polymer Membranes for Ultrafiltration," AIChE Annual Meeting, November 10-15, 1996, Chicago, IL.

2. Cohen, Y. , T. Faibish and M. Elimelech, "Flux Decline in Cross-Flow Filtration of Colloidal Silica", North America Membrane Society, Annual Meeting, Baltimore, Maryland, March, 1997.

3. Cohen, Y., "Graft Polymerized Ceramic Membranes", Gordon Research Conference on Membrane: Materials and Synthesis, New Hampshire, July, 1997.

4. Yoshida, W., Y. Cohen and Jeng-Deng J., "Graft Polymerization and Application to ceramic Supported Polymer Pervaporation membranes for VOC Removal”, AICHE Annual Meeting, Miami Beach, FL, November 15-20, 1998.

5. Rovira, M., F. Giralt and Y. Cohen, "Protein Fouling Reduction on ceramic Membranes via Graft Polymerization”, AICHE Annual Meeting, Miami Beach, FL, November 15-20, 1998.

6. Cohen, Y. and J-D. Jou, "Ceramic-Supported Polymer Pervaporation Membranes for VOC Removal," $10^{\text {th }}$ Annual Meeting of the North American Membrane Society, May 19-20, 1998, Cleveland, OH.

7. Cohen, Y., W. Yoshida and J-D, Jou, "CSP Membranes", invited poster presentation, DOE First Annual Environmental Management Science Program Scientific Workshop, Rosemont, IL, July 27-30, 1998.

8. Cohen, Y. Polymeric Resins for VOC Removal from Aqueous Systems, Volatile Organic Compounds (VOC) Recovery Seminar, EPA/625/R-99/005, ORD, EPA National Risk Management Research Laboratory, Sept 16-17, 1998.

9. Ron Faibish and Yoram Cohen, Crossflow Ultrafiltration of Oil-in-Water Microemulsions with Polymer-Modified Ceramic Membranes, 1999 AIChE Annual Meeting, October 31November 5, 1999, Dallas, TX

10. Wayne Yoshida, Jeng-dung Jou, Yong Liang, Yoram Cohen, Graft Polymerized Poly (vinyl acetate) and Poly(vinylpyrrolidone) Layers on Inorganic Substrates for Membrane Separations, 1999 AIChE Annual Meeting, October 31-November 5, 1999, Dallas, TX.

11. Yoram Cohen, Environmental Multimedia Distribution of Toxics (Mend-Tox), 1999 AIChE Annual Meeting, October 31-November 5, 1999, Dallas, TX.

12. Cohen, Y., Polymer at Solid Surfaces for Novel Membranes, invited presentation, Chemistry For a Cleaner Environment ( January 29-February 2, 2000, 2000), Santa Fe New Mexico.

13. Cohen, Y., DOE, Environmental Molecular Surface Laboratory, invited seminar, "Polymer Surfaces for Selective Separations", February 9, 1999. 


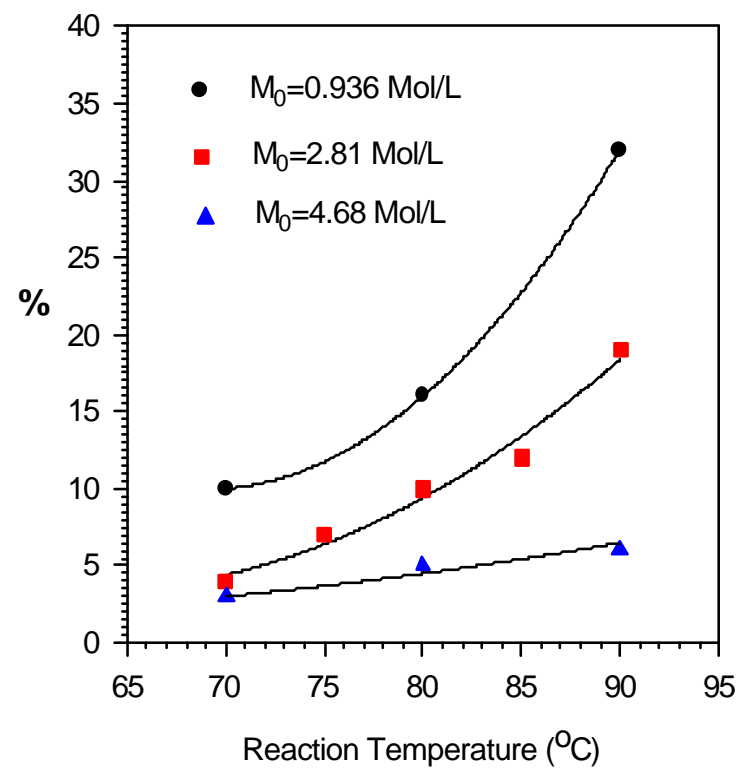

Figure 1. Percent contribution of polymer and graft e $t$ graft polymerization, to total graft yield for vinyl pyrvinyl pyrolidone graftpolymerization onto silica modified with vinyltrimethoxysilane.

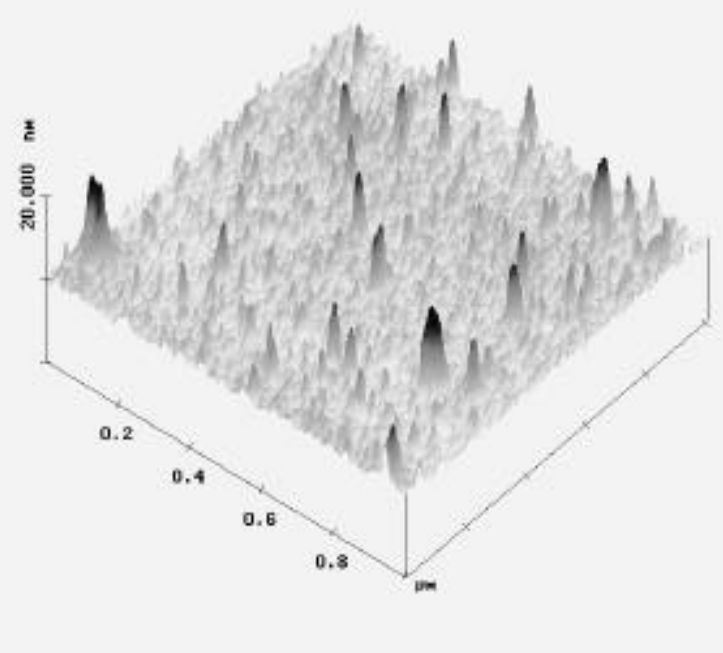

Figure 2 AFM surface images of silylated and PVP grafted silicon wafers (tapping mode).

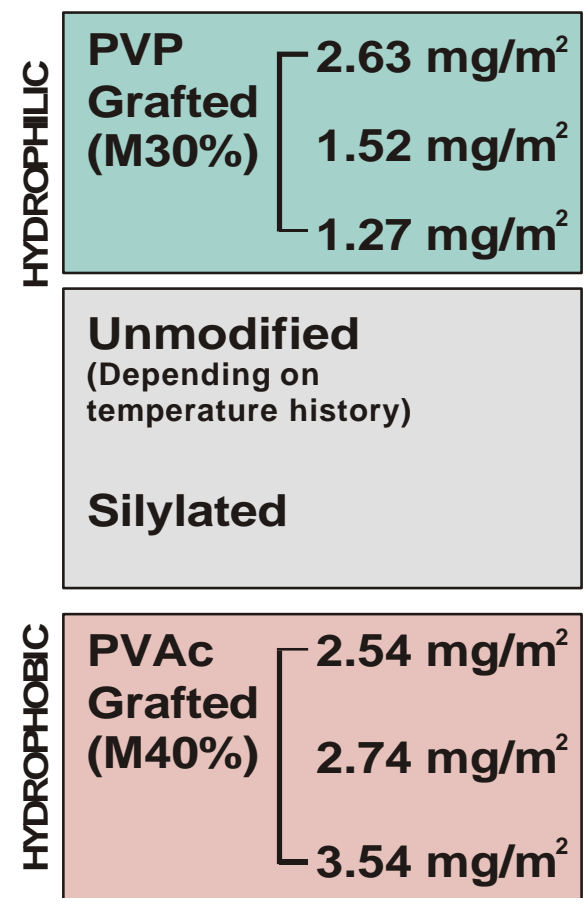

\subsection{2 \\ 40.84 \\ 59.00 \\ 27.83 \\ to \\ 67.53 \\ 66.54 \\ 69.51 \\ 72.00 \\ 69.26}

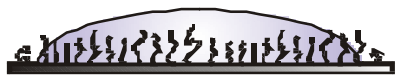
HYDROPHILIC SURFACE

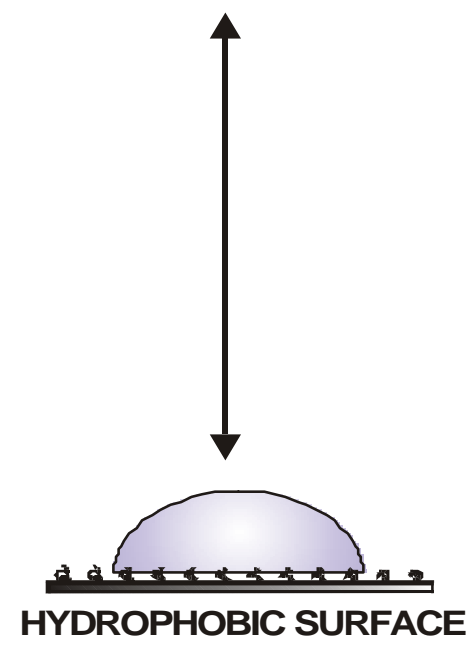

Substrate and Polymer Graft Yield

Contact angle $\left({ }^{\circ}\right)$

Figure 3. Contact angles for PVP and PVAc grafted onto a silicon wafer surface. Also shown are contact angels for unmodified and silylated surfaces. 


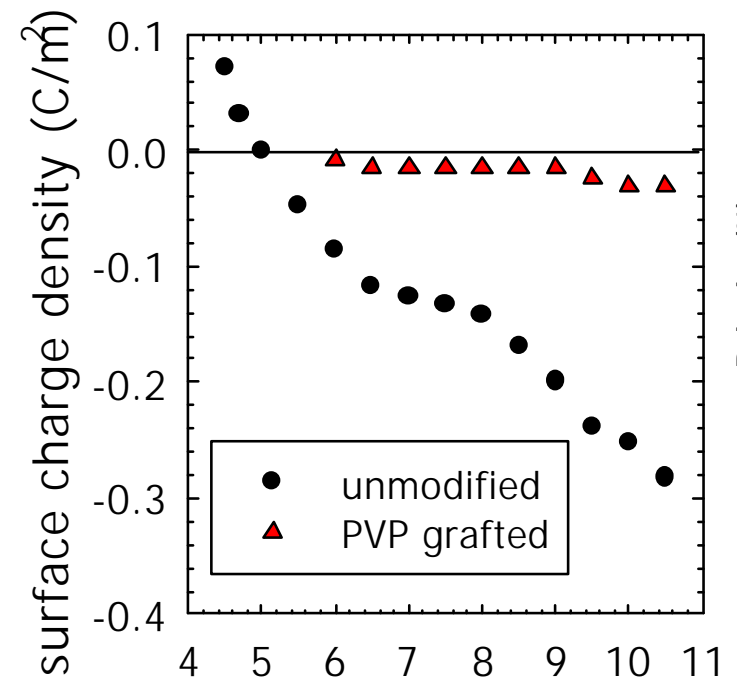

Figure 4. Surface hydroxyl masking on zirconia by grafted poly(vinyl pyrrolidone).

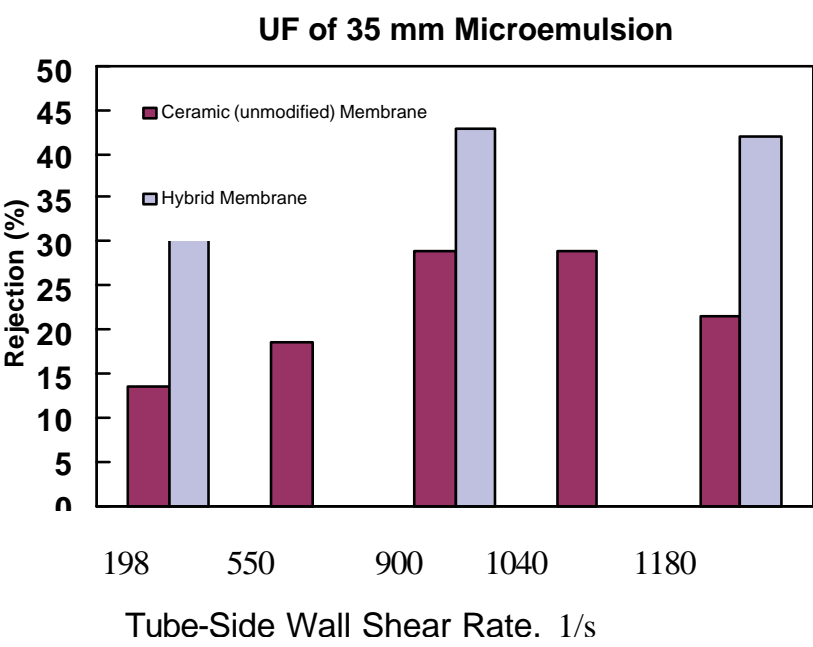

Figure 5. Ultrafiltration of oil-in-water microemulsion. (Transmembrane pressure $=64 \mathrm{kPa}$ ).

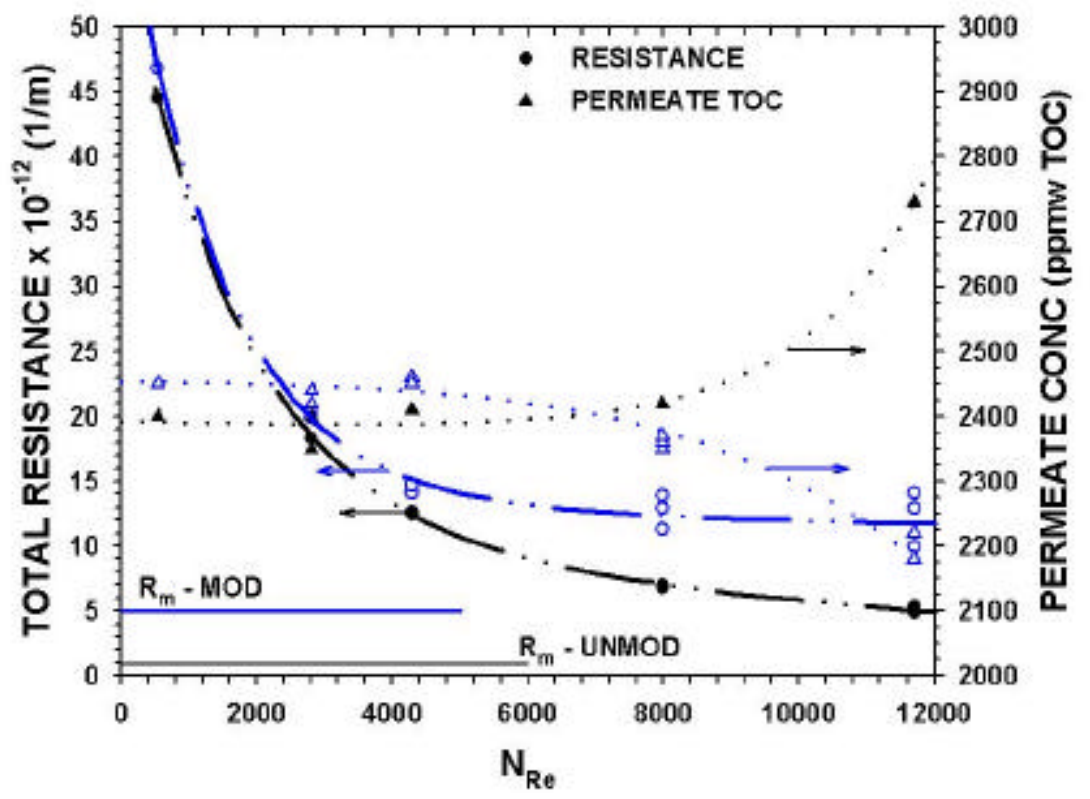

Figure 6. Total resistance and permeate concentration for UF of oil emulsion (41,000 ppm) using 410 $\mathrm{nm}$ native and grafted tubular silica membrane. 


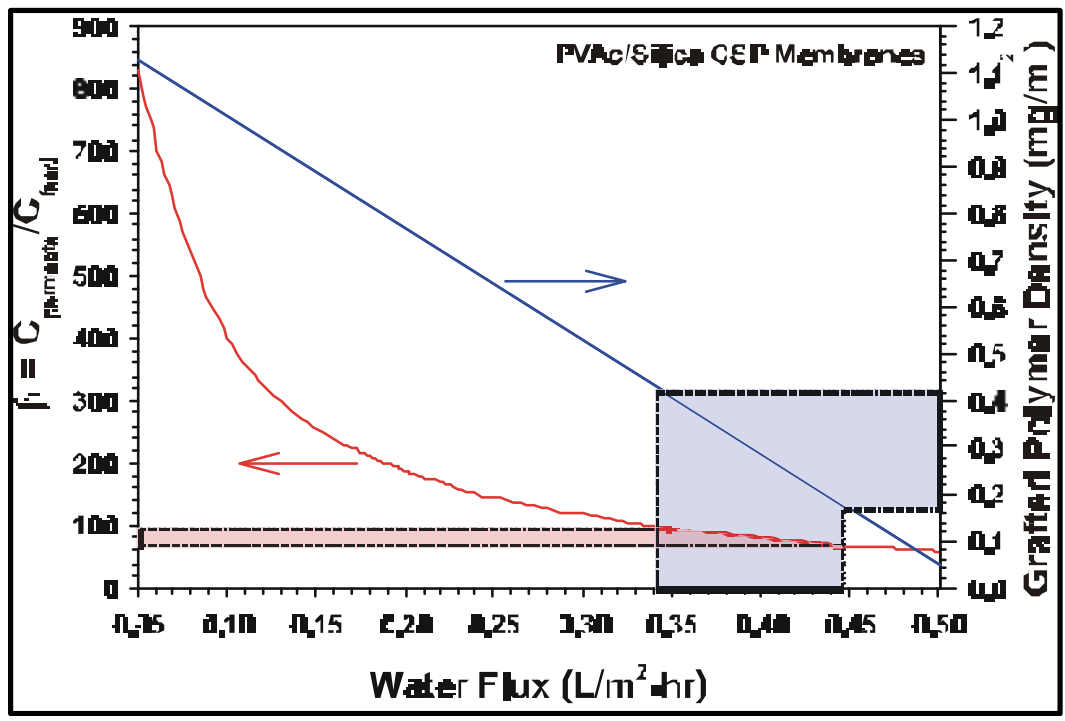

Figure 7. Predicted enrichment factor for trichloroethylene removal (from water) as a function of graft polymer density and water permeatiflux for a oly(vinyl acetate)-silica membrane. The shaded area represents the range of graft densities and enrichment factors acieved by Jou et al. ${ }^{[174]}$.

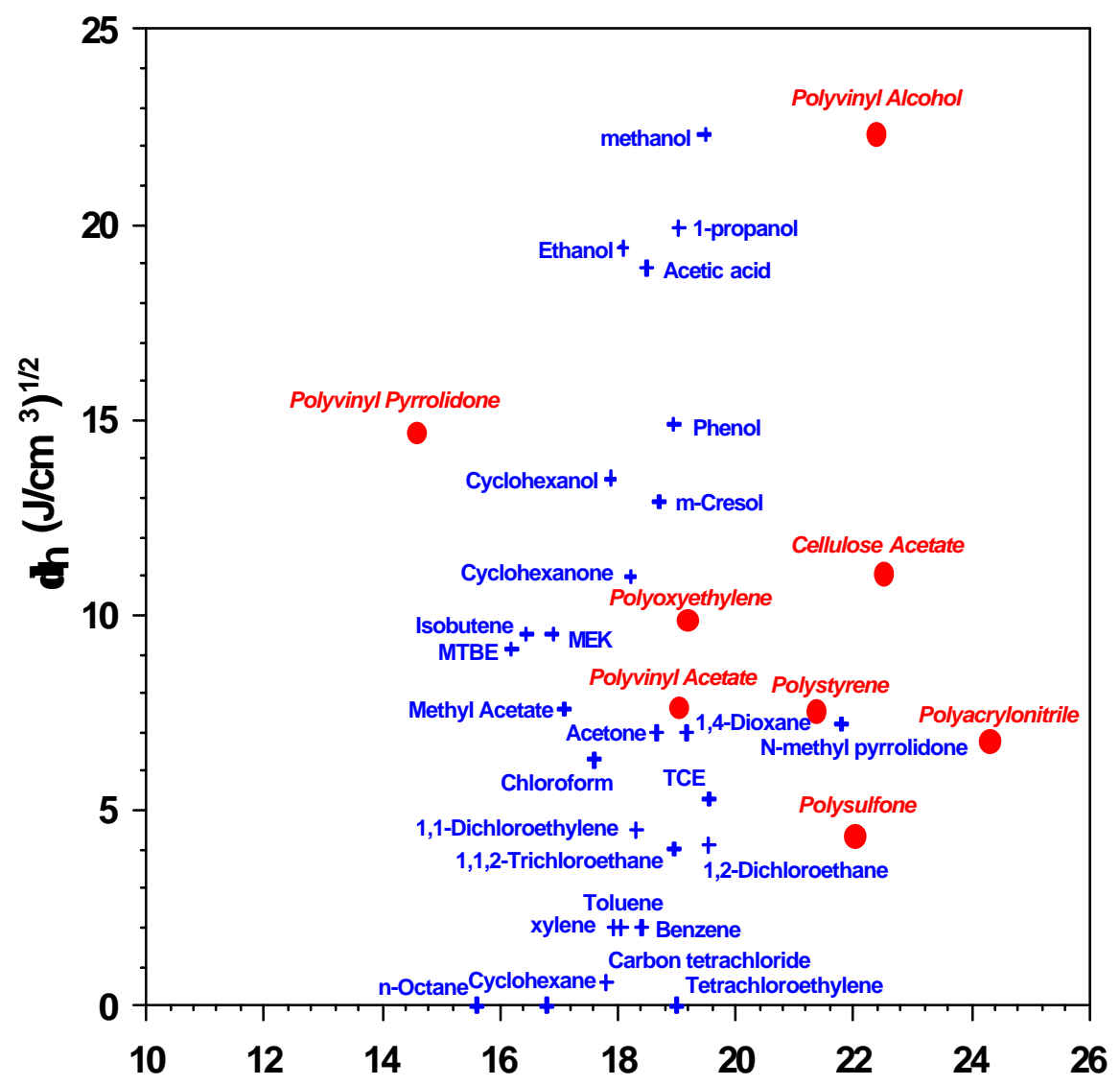

Figure 8. Hanson 2-D solubility parameter map. The large circles indicate a polymer and the crosses indicate solvent (or solute). 